Pacific Journal of Mathematic 


\title{
ON SYMMETRY OF SOME BANACH ALGEBRAS
}

\section{HORST LEPTIN}

\begin{abstract}
A Banach *-algebra is called symmetric, if the spectra of elements of the form $a^{*} a$ contain only nonnegative real numbers. Symmetric Banach *-algebras have a series of important properties, especially with respect to their representation theories. Here it is proved that tensoring with finite dimensional matrix rings preserves symmetry. As an application it is shown that the category of locally compact groups with symmetric $L^{1}$-algebras is closed under finite extensions.
\end{abstract}

In recent years there was a growing interest in the problem of symmetry of involutive Banach algebras. In particular very substantial progress has been made towards a solution of the problem of characterizations of such locally compact groups $G$ for which the group algebra $L^{1}(G)$ is symmetric. The most striking results in this direction are due to J. Jenkins, who first proved that the discrete " $a x+b$ "-group has a nonsymmetric algebra [3] and that the same holds for noncompact semisimple Lie groups [4] (independently this was also proved-but not published-by R. Takahashi). On the other hand, Hulanicki proved symmetry for discrete nilpotent groups (Studia Math. 35) and for class finite groups (Pacific J. Math. 18). Moreover, in Studia Math. $48 \mathrm{I}$ obtained the same resuits for connected nilpotent Lie groups of class 2 .

In [1] D. W. Bailey states a theorem (Theorem 2, p. 417) that a semi-direct product extension of a locally compact group $G$ with a finite group $F$ has a symmetric group algebra, if $G$ has. As (implicitely) in [2] and as in the present note this is reduced to the preservation of symmetry under tensoring with matrix algebras over $C$. His reduction of the $n \times n$-case to the $2 \times 2$-case is the same as ours, but his proof of the $2 \times 2$-case (Lemma 2, p. 415) contains a definitely false inequality for the spectral radius and thus is wrong.

Let $\Gamma$ be a locally compact group, $H$ a closed normal subgroup and let $G=\Gamma / H$ be the quotient group. Assume that we have a measurable cross section from $G$ into $T$. Then in [6] and [7] it was shown that $L^{1}(\Gamma)$ is isomorphic with a generalized $L^{1}$-algebra $L^{1}\left(G, L^{1}(H) ; T, P\right)$. Thus our result on groups will be a consequence of a more general one: Let $G$ be a finite group, $\mathscr{A}$ a Banach *algebra on which $G$ acts and let $P$ be a factor system of $G$ with values in the unitary multipliers of $\mathscr{A}$ (see [6], [7]). Then the 
generalized $L^{1}$-algebra $L^{1}(G, \mathscr{A} ; T, P)$ is symmetric, if $\mathscr{A}$ is symmetric. For commutative $\mathscr{A}$ and generalized $L^{1}$-algebras in the sense of [7] this has been proved by Glaser [2], using determinants. Our theorem will be a consequence of

THEOREM 1. Let $\mathscr{A}$ be an involutive Banach algebra and let $\mathscr{A}^{[n]}$ be the algebra of $n \times n$-matrices $x=\left(x_{i j}\right)$ over $\mathscr{A}, x_{i j} \in \mathscr{A}$, $i, j=1, \cdots, n$. If $\mathscr{A}$ is symmetric, then $\mathscr{A}^{[n]}$ is symmetric.

Proof. Assume at first that $\mathscr{A}$ contains an identity 1 and that $n=2$. Write $\mathscr{C}=\mathscr{A}^{[2]}$. We have to prove that for every $a=$ $\left(a_{i j}\right) \in \mathscr{C l}$ the left ideal

$$
\mathscr{L}=\mathscr{M}\left(1+a^{*} a\right)
$$

equals $\mathscr{l}$. (Here of course 1 is the unitmatrix and $a^{*}=\left(a_{i j}^{\prime}\right)$ with $\left.a_{i j}^{\prime}=a_{j i}^{*}\right)$. More generally consider the left ideal

$$
\mathscr{J}=\mathscr{M}(b * b+a * a)
$$

where $b=\left(b_{i j}\right)$ with $b_{21}=0, b_{11}=b_{22}=1$. The set $\mathscr{J}_{l k}$ of all $u \in \mathscr{A}$ for which there is an $x=\left(x_{i j}\right) \in \mathscr{J}$ with $u=x_{l k}$ plainly is a left ideal in $\mathscr{A}$. Since left multiplication with $\left(e_{2 j}\right), e_{11}=e_{22}=0, e_{12}=$ $e_{21}=1$, interchanges rows, we have $\mathscr{J}_{1 k}=\mathscr{J}_{2 k}=\mathscr{J}_{k}$ for $k=1,2$. Now $b^{*} b+a^{*} a=\left(z_{i j}\right) \in \mathscr{J}$. Hence $z_{11}=\mathbf{1}+a_{11}^{*} a_{11}+a_{21}^{*} a_{21} \in \mathscr{J}_{1}$ and $z_{22}=1+b_{12}^{*} b_{12}+a_{22}^{*} a_{22}+a_{12}^{*} a_{12} \in \mathscr{J}_{2}$. Clearly no nontrivial positive functional can vanish on $z_{i i}$ and hence on $\mathscr{J}_{i}$. This implies $\mathscr{J}_{i}=\mathscr{A}$ for $i=1,2$ (see [8], (4.7.14)). Now let $x=\left(x_{i j}\right) \in \mathscr{J}$ with $x_{11}=1$ and let $y=\left(y_{i j}\right)$ with $y_{12}=y_{22}=0$. Then $y x$ is in $\mathcal{J}$ and has $\left(y_{11}, y_{21}\right)$ as its first column. Similarly one shows that $\mathscr{J}$ contains elements with given second column.

Now consider again $\mathscr{L}$ and let $x \in \mathscr{L}$ with $x_{11}=1$. Multiplying $x$ from the left by $f=\left(f_{i j}\right), f_{11}=1, f_{\imath j}=0$ otherwise, we may assume that $x_{21}=x_{22}=0$. Define $b=\left(b_{i j}\right)$ by $b_{11}=b_{22}=1, b_{12}=-x_{12}$ and $b_{21}=0, c=a b$. We have $\mathscr{M}\left(b^{*} b+c^{*} c\right)=\mathscr{M} b^{*}\left(1+a^{*} a\right) b=\mathscr{L} b$. It follows that $\mathscr{J}=\mathscr{C}\left(b^{*} b+c^{*} c\right)$ contains $x b=f$. From this one derives easily that every matrix with second column equal to zero is contained in $\mathscr{J}$. But we know already that arbitrary second columns occur in elements from $\mathscr{F}$. This implies that $\mathscr{J}$ contains all matrices, i.e., $\mathscr{J}=\mathscr{H}$. Since $b$ is regular, also $\mathscr{L}=\mathscr{M}$.

2. Now let $n$ be arbitrary. By induction it follows that all $\mathscr{A}^{\left[2^{l}\right]}$ are symmetric. For $n \leqq 2^{l}, \mathscr{A}^{[n]}$ can be considered as sub-

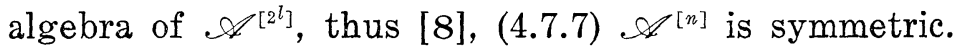

3. If $\mathscr{A}$ has no unit, the algebra $\mathscr{A}=(1) \oplus \mathscr{A}$ is symmetric 
[8], (4.7.9) and contains $\mathscr{A}$. It follows that $\tilde{\mathscr{A}}^{[n]}$ is symmetric and contains $\mathscr{A}^{[n]}$, which in turn is symmetric. Thus Theorem 1 is proved.

Now let $G$ be a locally compact group which acts on the involutive Banach algebra $\mathscr{A}$. This means that there exists a mapping $T$ of $G$ into the group Aut $(\mathscr{A})$ of isometric *automorphisms of $\mathscr{A}$, such that $T_{x} T_{y} T_{x y}^{-1}$ is contained in the adjoint algebra $\mathscr{A}^{b}$ of $\mathscr{A}(=$ algebra of "double centralizers") [7]. If $P$ is a unitary 2-cocycle one can form the generalized $L^{1}$-algebra $L^{1}(G, \mathscr{A} ; T, P)$, see [6].

THEOREM 2. If $G$ is finite and $\mathscr{A}$ is symmetric, then $L^{1}(G, \mathscr{A} ; T, P)$ is symmetric.

Proof. Let $n$ be the order of $G$. The matrix algebra $\mathscr{A}^{[n]}$ acts naturally on the $n$-fold direct sum $n \mathscr{A}=\mathscr{A} \oplus \cdots \oplus \mathscr{A}$. On the other hand, identifying $\mathscr{L}=L^{1}(G, A ; T, P)$ and $n \mathscr{A}$ as Banach spaces convolution also defines an action of $\mathscr{L}$ on $n \mathscr{A}$. The formula for the convolution product in $\mathscr{L}$ shows, that for $f \in \mathscr{L}$ the convolution operator on nef corresponds to the matrix

$$
M(f)=\left(P_{y x^{-1}, x} T_{x^{-1}} f\left(y x^{-1}\right)\right)_{x, y} .
$$

Thus $f \rightarrow M(f)$ is an isomorphism of $\mathscr{C}$ into $\mathscr{A}^{[n]}$. Also $M\left(f^{*}\right)=$ $M(f)^{*}$ holds. It suffices to prove this only for functions of the form $f(x)=\delta_{x, z} a$ with fixed $a \in \mathscr{A}$ and $z \in G$. For such a function the claim is equivalent with the identity

$$
\left(P_{z, y} T_{y^{-1}} a\right)^{*}=P_{z^{-1, z y}} T_{(z y)^{-1}} P_{z, z^{-1}}^{0} T_{z} a^{*}
$$

which can be proved by using the definitions and relations of [6], esp. (1.2), (1.3) and the definition of $T_{x, y}$ on p. 595 .

It follows that $\mathscr{L}$ can be considered as a subalgebra of $\mathscr{A}^{[n]}$. Hence by Theorem 1 and [8], (4.7.7) symmetry of $\mathscr{A}$ implies that of $\mathscr{L}$.

THEOREM 3. Let $G$ be a locally compact group and let $H$ be a closed subgroup of finite index. Then $L^{1}(G)$ is symmetric if and only if $L^{1}(H)$ is symmetric.

Proof. Since $H$ is open in $G, L^{1}(H)$ is a subalgebra of $L^{1}(G)$ and consequently is symmetric if $L^{1}(G)$ is.

Now let $L^{1}(H)$ be symmetric. The intersection $H_{0}$ of the finite number of conjugate subgroups $x H x^{-1}$ of $H$ in $G$ is closed, normal and also of finite index in $G$. Moreover, $L^{1}\left(H_{0}\right)=\mathscr{A}$ as we have seen is symmetric. But $L^{1}(G) \cong L^{1}\left(G / H_{0}, \mathscr{A} ; T, P\right)$ ([6], Satz 5). Therefore, Theorem 3 follows now from Theorem 2 . 


\section{REFERENCES}

1. D. W. Bailey, On symmetry on certain group algebras, Pacific J. Math., 24 (1968), 413-419.

2. W. Glaser, Symmetrie von verallgemeinerten L1-Algebren, Arch. Math., 20 (1969), 656-660.

3. J. Jenkins, An amenable group with a nonsymmetric group algebra, Bull. Amer. Math. Soc., 75 (1969), 45-47.

4. - Nonsymmetric group algebras, to appear in Studia Math.

5. - Manuscript, April 1973.

6. M. Leinert, Beiträge zur Theorie der verallgemeinerten $L^{1}$-Algebren, Arch. Math., 21 (1970), 594-600.

7. H. Leptin, Verallgemeinerte $L^{1}$-Algebren und projektive Darstellungen lokal kompalter Gruppen, Inventiones Math., 3 (1967), 257-281, ibid., 4 (1967), 68-86.

8. C. E. Rickart, General Theory of Banach Algebras, Van Nostrand, New York, 1960. 


\section{PACIFIC JOURNAL OF MATHEMATICS}

\section{EDITORS}

RICHARD ARENS (Managing Editor)

University of California

Los Angeles, California 90024

R. A. Beaumont

University of Washington

Seattle, Washington 98105
J. DugundJI

Department of Mathematics

University of Southern California

Los Angeles, California 90007

D. Gilbarg and J. Milgram

Stanford University

Stanford, California 94305

\section{ASSOCIATE EDITORS}

E. F. BECKENBACH

B. H. NeUMANN

F. WOLF

K. YosHIDA

\section{SUPPORTING INSTITUTIONS}

UNIVERSITY OF BRITISH COLUMBIA

CALIFORNIA INSTITUTE OF TECHNOLOGY

UNIVERSITY OF CALIFORNIA

MONTANA STATE UNIVERSITY

UNIVERSITY OF NEVADA

NEW MEXICO STATE UNIVERSITY

OREGON STATE UNIVERSITY

UNIVERSITY OF OREGON

OSAKA UNIVERSITY
UNIVERSITY OF SOUTHERN CALIFORNIA

STANFORD UNIVERSITY

UNIVERSITY OF TOKYO

UNIVERSITY OF UTAH

WASHINGTON STATE UNIVERSITY

UNIVERSITY OF WASHINGTON

$\stackrel{*}{*} \stackrel{*}{*} \stackrel{*}{*}$ AMERICAN MATHEMATICAL SOCIETY




\section{Pacific Journal of Mathematics}

\section{Vol. 53, No. $1 \quad$ March, 1974}

Martin Bartelt, Strongly unique best approximates to a function on a set, and a finite

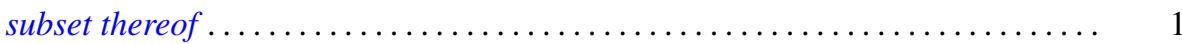

S. J. Bernau, Theorems of Korovkin type for $L_{p}$-spaces $\ldots \ldots \ldots \ldots \ldots \ldots \ldots \ldots \ldots$

S. J. Bernau and Howard E. Lacey, The range of a contractive projection on an

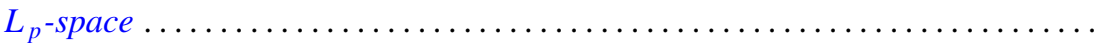

Marilyn Breen, Decomposition theorems for 3-convex subsets of the plane ......... Ronald Elroy Bruck, Jr., A common fixed point theorem for a commuting family of

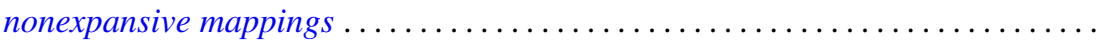

Aiden A. Bruen and J. C. Fisher, Blocking sets and complete $k$-arcs . . . . . . . 73

R. Creighton Buck, Approximation properties of vector valued functions . ......... 85

Mary Rodriguez Embry and Marvin Rosenblum, Spectra, tensor products, and

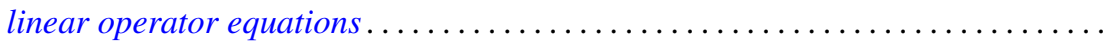

Edward William Formanek, Maximal quotient rings of group rings . . . . . . . . . 109

Barry J. Gardner, Some aspects of T-nilpotence . . . . . . . . . . . . . . . 117

Juan A. Gatica and William A. Kirk, A fixed point theorem for $k$-set-contractions

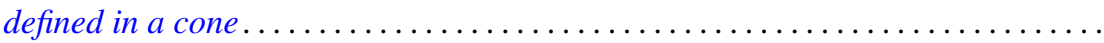

Kenneth R. Goodearl, Localization and splitting in hereditary noetherian prime

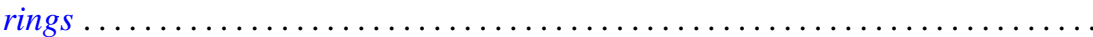

James Victor Herod, Generators for evolution systems with quasi continuous

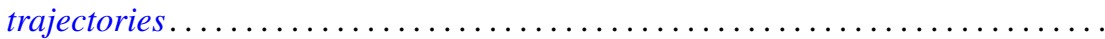

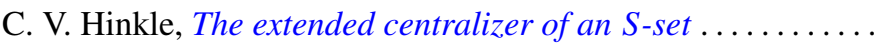

I. Martin (Irving) Isaacs, Lifting Brauer characters of p-solvable groups . . .

Bruce R. Johnson, Generalized Lerch zeta function ...........

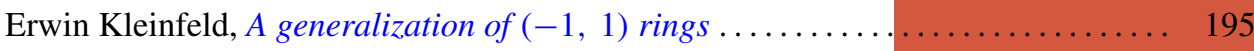

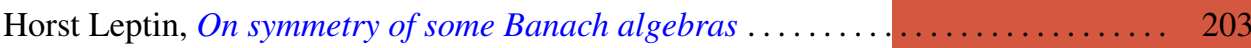

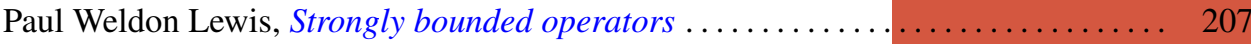

Arthur Larry Lieberman, Spectral distribution of the sum of self-adjoint

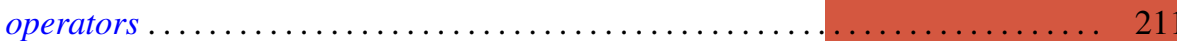

I. J. Maddox and Michael A. L. Willey, Continuous operators on paranormed spaces and matrix transformations

James Dolan Reid, On rings on groups ........................... 229

Richard Miles Schori and James Edward West, Hyperspaces of graphs are Hilbert cubes.

William H. Specht, A factorization theorem for p-constrained groups ...

Robert L Thele, Iterative techniques for approximation of fixed points of certain nonlinear mappings in Banach spaces ...............

Tim Eden Traynor, An elementary proof of the lifting theorem

Charles Irvin Vinsonhaler and William Jennings Wickless, Completely decomposable groups which admit only nilpotent multiplications .

Raymond O’Neil Wells, Jr, Comparison of de Rham and Dolbeault cohomology for

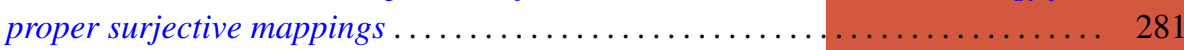

David Lee Wright, The non-minimality of induced central representations . . . . . 301 